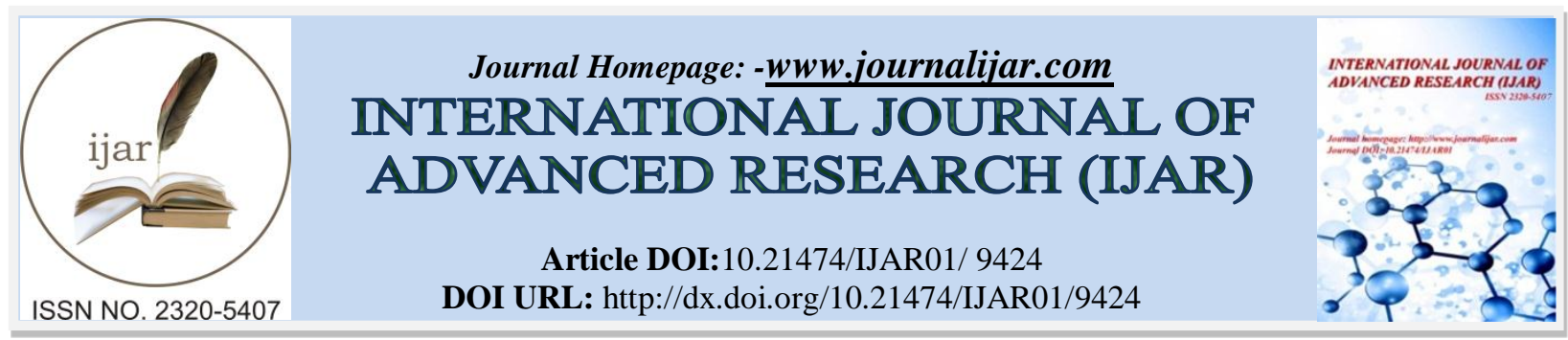

RESEARCH ARTICLE

\title{
BILATERAL UNIPORTAL VIDEO-ASSISTED THORACOSCOPIC SURGERY SYMPATHECTOMY FOR MANAGING RAYNAUD'S DISEASE.
}

Yasser Aljehani, Rahma ShahBahai, Alaa Turkistani.

Thoracic Surgery Division, Department of Surgery, King Fahad Hospital of the University. College of Medicine, Imam Abdulrahman Bin Faisal University, Dammam, Saudi Arabia.

\section{Manuscript Info}

Manuscript History

Received: 22 May 2019

Final Accepted: 24 June 2019

Published: July 2019

Key words:-

Raynaud's disease, Uniportal

sympathectomy, VATS.

\section{Abstract}

Video-Assisted Thoracoscopic Surgery (VATS) indication has expanded over the years to include medical treatment refractory to standard medical therapy. The introduction of bilateral uniportal VATS sympsthectomy (uniVATS) in the treatment of Raynaud's disease (RD) is highlighted in such case. The results are promising and the pre-operative testing is emphasized.

Copy Right, IJAR, 2019,. All rights reserved.

\section{Introduction:-}

Raynaud's disease is an intermittent reduction of blood supply incorporated with vasospasm in the digital arteries of the upper extremities which can be triggered by cold and emotional stress [1]. Pain in association with pallor and/or cyanosis are the usual clinical complaint by the patients. There is no definitive treatment method to cure the symptoms. VATS sympathectomy has been used in current years to relieve the symptoms of RD.

We present a case of a young lady, known case of rheumatoid arthritis presented to our thoracic surgery clinic with $\mathrm{RD}$ affecting her both hands and was managed by bilateral uniVATS sympathectomy.

\section{Case description:}

A 35-years old lady presented to thoracic surgery clinic with bilateral RD affecting her both hands. This patient has been diagnosed with rheumatoid arthritis for more than 10 years. However, she was diagnosed with Raynaud's disease since then and it was getting progressive. On examination, both hands revealed bluish hands with a sluggish capillary refill in her fingernails. Her hands feel subjectively cold. Pulse oximeter could not detect a pulse wave. Her investigation showed antinuclear antibody $>1280 \mathrm{IU} / \mathrm{ml}$, Rheumatoid factor $108.7 \mathrm{IU} / \mathrm{ml}$ and Positive antiribonucleoprotein. Normal Complete blood count and C3\& C4 complement. Negative anti-Beta-2 Glycoprotein-1 Antibodies, anti-double-strand DNA antibodies, anticardiolipin antibodies, anti-Scl-70 antibodies, anti-SSA antibodies, anti-SSB antibodies or anti-RNP antibodies. For treatment, she didn't respond to medical therapy including calcium channel blockers. Therefore, she was offered sympathectomy. The patient was evaluated by anesthesiologist and they decided to do a trial of Stellate ganglion block as a diagnostic procedure using local anesthetic injection (total of $5 \mathrm{ml}$ of $0.25 \%$ bupivacaine mixed with one in 200.000 epinephrine) for the right side only as it is commonly will affect both sides and to prevent bilateral phrenic nerve or recurrent laryngeal nerve paralysis. In recovery room patient developed temporary right sided Horner syndrome which was expected. Also, she gets some changes at the right-hand temperature as it was $25^{\circ} \mathrm{C}$ before the procedure and $34{ }^{\circ} \mathrm{C}$ after it which consider 2nd-degree change and it is significant. The color of fingers changed from bluish to pinkish. All of these changes indicate a successful block and lasted for 3-4 hours after the procedure. The patient underwent bilateral 
uniVATS Sympathectomy almost 1-cm incision. An incision was done at 7th intercostal space (ICS) posterior axillary line in which sympathetic chain was cut below 3rd and 4th levels. Post-operative course was unremarkable. In follow up, symptoms disappeared and the patient improved and satisfied.

\section{Discussion:-}

The etiology of RD has not been fully explained in the current literature [2]. Pain in association with pallor and/or cyanosis is the usual clinical complaint by the patients. The prevalence of RD is about 3\%-4\% with preponderance among women [2]. Although RD might lead to digital ischemia and gangrene or tissue loss; these complications are rare, and usually it has a benign prognosis $[2,3]$. The management starts with medical treatment but there is no definitive treatment method to cure the symptoms [4]. Yet, conservative management such as reduction of emotional stress, protection from cold and quitting smoking in addition to medical therapy are known to have little effect in minimizing the signs and symptoms related to Raynaud's phenomenon [4]. Available surgical options are balloon angioplasty, venous or arterial grafting and sympathectomy demonstrating a variable result [5]. In the era of thoracoscopic uniVATS, this is a valid option especially if combined with pre-operative assessment such as sympathetic ganglion block and objectively documenting the response. VATS sympathectomy reduces the peripheral vascular resistance, thus increasing the blood flow to the peripheral vascular system [5]. However, it has been used in current years to abolish the symptoms incorporated to Raynaud's disease cases including frequent and severe attacks, tissue damages such as non-healing digital ulceration and necrosis, as well as dystrophic changes occur in the extremities regardless of the adequate medical treatment [5-7].

\section{Conclusion:-}

In selected case of Raynaud's disease, the surgical option is even less with bilateral uniVATS sympathectomy especially if the trail of the stellate ganglion is of a positive response with a minimal scar and no complication.

\section{References:-}

1. Lambova SN, Müller-Ladner U. New lines in therapy of Raynaud's phenomenon. Rheumatol Int. (2009);29:355-5. [PubMed]

2. Fardoun MM, Nassif J, Issa K, et al. Raynaud's phenomenon: a brief review of the underlying mechanisms. Front Pharmacol. 2016;7:438

3. Karapolat S, Turkyilmaz A, Tekinbas C. Effects of Endoscopic Thoracic Sympathectomy on Raynaud's Disease. Journal of Laparoendoscopic \& Advanced Surgical Techniques. 2018;

4. Sinnathurai P, Schrieber L. Treatment of Raynaud phenomenon in systemic sclerosis. Internal Medicine Journal. 2013;43(5):476-483

5. Landry G. Current medical and surgical management of Raynaud's syndrome. Journal of Vascular Surgery. 2013;57(6):1710-1716.

6. Coveliers H, Hoexum F, Rauwerda J, Wisselink W. Endoscopic thoracic sympathectomy for upper limb ischemia. A 16 year follow-up in a single center. The Surgeon. 2016;14(5):265-269.

7. Dao T, Amaro-Driedger D, Mehta J. Successful treatment of raynaud's syndrome in a lupus patient with continuous bilateral popliteal sciatic nerve blocks: A case report. Local and Regional Anesthesia. 2016 Jun 15;9:35-37. Available from, DOI: 10.2147/LRA.S97111. 